\title{
Reducing Mortality in Critically Ill Patients
}

\author{
Giovanni Landoni, Marta Mucchetti, Alberto Zangrillo, Rinaldo Bellomo (Editors). \\ Springer, 2015; ISBN 978-3-319-17514-0
}

\author{
Carmen Hrymak, MD
}

Received: 11 September 2015/Accepted: 6 October 2015/Published online: 19 October 2015

(C) Canadian Anesthesiologists' Society 2015

Critical care physicians strive to reduce patient mortality through appropriately applying evidence-based medicine to clinical practice. This takes both skill in critical appraisal of the literature and dedication of time for a thorough review. Even compelling randomized-controlled trials (RCTs) are not always replicated in subsequent studies and applying results to everyday care is a complex task. Through the use of democracy-based medicine, Reducing Mortality in Critically Ill Patients identifies nonsurgical interventions that have either a positive or a negative effect on mortality in critically ill patients.

The process of democracy-based medicine is described in the first chapter and sets the stage for the entire book. This unique approach to evidence deserves some description. First, a systematic review was performed to capture all nonsurgical interventions evaluated by multicentre RCTs. The articles had to be published in peer-reviewed journals and needed to show that the interventions have a statistically significant impact on mortality in critically ill patients. Next, an international consensus conference of experts-participation from 555 physicians in 61 countries - combined with web-based worldwide surveys, was used to validate the findings. Fifteen interventions impacting mortality were identified, and the editors dedicate the remainder of the book to the details of these interventions. Information from this consensus is also published in Critical Care Medicine. ${ }^{\mathrm{A}}$

C. Hrymak, MD ( $\bowtie)$

Department of Internal Medicine, Section of Critical Care,

University of Manitoba, Health Sciences Centre, Winnipeg, MB, Canada

e-mail: umhrymak@myumanitoba.ca
Several authors contributed to each chapter, many of whom were involved directly with the primary literaturethe result is a well-informed product. The variety in authorship does not make the book seem disjointed. Chapters flow with a similar style and the entire book is easy to read-a tribute to the experienced editorial team. Each chapter is neatly divided into general principles and main evidence, and depending on the topic, pathophysiological principles, pharmacologic properties, and therapeutic use. Clinical summary tables reiterate conclusions drawn within the text, and references compiled at the end of each chapter facilitate searching for primary literature and updates.

Part I, titled "Interventions that Reduce Mortality", consists of six chapters that cover such topics as noninvasive ventilation, lung-protective ventilation in acute respiratory distress syndrome, tranexamic acid in trauma patients, albumin use in liver cirrhosis, and daily interruption of sedatives. The layout is consistent but not rigid from chapter to chapter, for instance, sections are expanded for topics deserving of a more in-depth description. The result is more than just a summary of the literature but a comprehensive review of each subject. Helpful illustrations are scattered throughout the book. In addition, practical descriptions of applying the information are included where indicated, for example, calculation of predicted body weight in lung-protective ventilation and an in-depth physiologic description of prone positioning.

Part II, titled "Interventions that Increase Mortality", consists of eight chapters, each dedicated to an individual

\footnotetext{
$\overline{\mathrm{A}}$ Landoni $G$, Comis $M$, Conte $M$, et al. Mortality in multicenter critical care trials: an analysis of interventions with a significant effect. Crit Care Med 2015; 43: 1559-68.
} 
intervention. Interventions showing increased mortality are: tight glycemic control, hydroxyethyl starch, growth hormone, diaspirin cross-linked hemoglobin and other blood substitutes, supranormal oxygen delivery, beta 2 agonists in acute respiratory distress syndrome, high frequency oscillatory ventilation, and glutamine supplementation. Reviews, summaries, and clinical indications are provided.

Evidence-based medicine continues to evolve and can change considerably in the time it takes to publish material. The systematic review for this book was completed in June 2013. The editors put forth a commendable effort to ensure the book is as current as possible by including multiple references from 2015 and by dedicating a third section, Part III, to updated medicine, including therapeutic hypothermia. This summarized literature will continue to serve as a solid foundation and useful reference upon which to build in future.

Specialists from areas of medicine that care for patients with critical illness will enjoy this book, especially those working in the intensive care unit. Experienced physicians will be comfortable with much of the subject matter and familiar with the landmark trials. Furthermore, the book provides useful clinical summaries and practical application points that even physicians well versed in the literature will appreciate. Residents and fellows will benefit from the organized format and detailed pathophysiologic explanations.

In summary, Reducing Mortality in Critically Ill Patients, while only 140 pages in length, is a high-yield quality compilation of interventions that influence mortality. In addition, a large group of physicians identified the interventions through a democracy-based medicine approach. Readers will gain more confidence in the application, or appropriate avoidance, of the 15 major interventions covered in this text.

Funding sources None

Conflicts of interest None declared. 